\title{
Perfil das escolas de posturas implantadas no Brasil
}

\author{
Profile of the back school implanted in Brazil
}

\author{
Carla Bianca da Silva Santos ${ }^{1}$; Demóstenes Moreira ${ }^{2}$
}

Resumo

Este artigo retrata a implantação da "Escola de Postura" no Brasil e os procedimentos por ela propostas utilizada como forma de prevenção e tratamento das algias da coluna, desde sua criação, em 1969. No Brasil, os resultados de vários estudos realizados acerca do assunto trazem resultados variados o que pode por em dúvida a questão de sua eficácia. Busca-se fixar o perfil da escola, devido às necessidades de se conhecer os efeitos deste programa, e, assim, efetuou-se uma revisão da literatura acerca da implantação e difusão da "Escola de Postura" no Brasil, as diversas modificações e adaptações ocorridas ao longo dos anos, os recursos utilizados, os locais em que ela tem sido implantada e, também, os resultados apresentados por cada uma delas com relação a sua eficácia em pacientes com lombalgia crônica.

Palavras-chave: Algias da coluna. Escola de Postura. Lombalgia.

\begin{abstract}
This essay presents the establishment of the "Back School" in Brazil and how it has been used as a prevention and treatment form of the spine pains since its foundation in 1969. In Brazil, the results of various studies on the subject have varied consequences which can question its effectiveness. In search of a profile and the need to know the effects on this program, it was necessary to review the literature on the establishment and spreading of the "Back School" in Brazil, the several changes and adjustments that have occurred for years, the resources used, where this type of School has been established, and also the results presented by each one of them concerning their effectiveness in patients with chronic low back pain.
\end{abstract}

Key words: Back pain. Back School. Low back pain.

\footnotetext{
1 Mestre em Ciências da Saúde pela Universidade de Brasília (UnB). Fisioterapeuta. Professora no curso de Fisioterapia da Faculdade de Ciências Biomédicas de Cacoal/RO (FACIMED). E-mail: carlabyanca@hotmail.com

2 Mestre e Doutor em Ciências da Saúde pela Universidade de Brasília (UnB).Fisioterapeuta do HRAN/Brasília, DF.
} 


\section{Introdução}

Não são recentes os problemas que afligem os homens no que refere as dores lombares. Segundo Snook (1978), existem relatos apresentados pelos egípcios, há 5000 anos e, com o avanço da tecnologia e da industrialização, o problema vem se agravando cada vez mais. Países industrializados são os que possuem os maiores índices desta morbidade, em torno de 70 \% (SILVA; NEIVA; VALLE, 2004).

No Brasil, estima-se que aproximadamente 10 milhões de pessoas ficam incapacitadas por causa de dores lombares e que pelo menos $70 \%$ da população brasileira sofrerão pelo menos um episódio de dor na vida (TEIXEIRA, 1999).

Devido aos níveis epidêmicos que o problema atingiu, este passou a ser um tema trabalhado por muitos autores no intuito da busca de uma solução para o mesmo, um dos principais caminhos propostos foi um trabalho voltado para a educação postural. Segundo Braccialli e Vilarta (2000), a educação postural tem como finalidade de capacitar a pessoa a proteger-se ativamente de lesões durante seus movimentos nas suas atividades de vida diária e profissional, seja no plano estático ou dinâmico.

Nesse sentido, surgiu em 1969, na Suécia, a "Back School", um programa idealizado pela fisioterapeuta Mariane Forssel (HEYMANS et al., 2005). Esse programa tinha como principal objetivo ensinar as pessoas a gerenciar a sua própria saúde, "melhorando a capacidade do individuo em cuidar de sua coluna através de explicações sobre lombalgia e quais atitudes pode o próprio individuo tomar em relação a essa patologia"(FORSSELL, 1981), dandolhes noções de anatomia e biomecânica da coluna. Assim, elas aprendiam sobre as posturas corretas durante a execução de atividades corriqueiras e laborativas, realizavam práticas de relaxamento e fortalecimento, e podiam depois evitar posturas que pudessem, de alguma forma, comprometer o seu sistema músculo esquelético.

A "Back School" espalhou-se por diversos países do mundo e em cada um deles o programa foi adaptado às necessidades locais. No Brasil, ele chegou em 1972, três anos após sua idealização, foi implantado no Hospital do Servidor Público Estadual de São Paulo pelo médico José Knoplich.

Posteriormente, o programa foi implantado e desenvolvido por diversas instituições brasileiras, como veremos a seguir. Assim, identificaremos o perfil de cada uma delas, e também analisar os resultados alcançados por elas, que é o principal objetivo deste trabalho.

\section{Metodologia}

A revisão bibliográfica foi realizada por meio de portais de busca da internet que possuem bases de dados de pesquisa em saúde tais como MEDLINE e Literatura Latino-Americana e do Caribe em Ciências da Saúde (LILACS). Como critério de busca, utilizaram-se combinações de palavraschave como algias da coluna, escola de postura e lombalgia. Dessa forma, foram encontradas sobre o assunto duas (02) teses de mestrado, quatro (04) artigos internacionais e mais oito (08) artigos nacionais que relatam experiências com a escola de postura.

Uma revisão desta natureza requer uma estratégia de busca, e é necessário o estabelecimento de critérios de inclusão e exclusão dos artigos e, acima de tudo, uma análise criteriosa da qualidade da literatura selecionada, a identificação dos conceitos importantes, a comparação das análises estatísticas apresentadas. Também cabe verificar se a literatura informa uma relação entre a intervenção proposta, e se ela aponta ainda problemas/questões que necessite de novos estudos.

Entre todos os artigos encontrados a respeito do assunto, foram excluídas as duas teses de mestrado, os quatro artigos internacionais, pois estávamos interessados em resultados de escolas implantadas no Brasil, e mais dois artigos nacionais por não retratarem com clareza a descrição de hipóteses ou objetivos do estudo, as características dos pacientes 
incluídos, os quais eram as intervenções de interesse e os principais achados do estudo.

Os seis artigos selecionados foram lidos integralmente, no intuito de verificar se preenchiam todos os critérios. Outro fator fundamental e decisivo foi o apoio dado pelas instituições a estes programas, pois este pode determinar a longevidade dos mesmos e o envolvimento da equipe multidisciplinar para que eles obtivessem sucesso. A respeito, é preciso levar em consideração que são poucas instituições que implantaram este programa e conseqüentemente existem poucas publicações sobre o assunto no Brasil.

\section{Resultados}

No intuito de discutir os resultados encontrados pelas escolas de posturas selecionadas neste estudo, inicialmente faremos uma breve descrição de cada uma delas divididas pelas respectivas instituições nas quais foram realizadas e, posteriormente, uma comparação dos principais resultados.

Faculdade de Medicina da Universidade de São Paulo (FMUSP)

A Faculdade de Medicina da Universidade de São Paulo (FMUSP) iniciou sua proposta de "Back School", em 1994, denominada "Escola das Costas", cujo programa foi dirigido para pacientes com dor em todos os segmentos da coluna (cervical, dorsal e/ou lombar).

Esta escola foi implantada pela Divisão de Medicina Física do Hospital das Clínicas da Universidade de São Paulo, e nela foram estudados 32 pacientes. Foram trabalhadas atividades teóricas voltadas aos assuntos de anatomia e biomecânica da coluna vertebral, epidemiologia, alimentação equilibrada, importância da vida social, ergonomia, cisnesiologia, primeiros socorros e também atividades práticas como alongamento, dança, ergonomia prática e adequação do ambiente de trabalho (CHUNG, 1996).
Neste estudo, foram incluídas somente pessoas com dor crônica de coluna, estes pacientes foram acompanhados por uma equipe multidisciplinar envolvida na escola de coluna tais como: médico fisiatra, fisioterapeuta, educador físico, terapeuta ocupacional, nutricionista, psicólogo e assistente social.

Também no ano de 1994 o Instituto de Ortopedia e Traumatologia organizou uma escola de postura, da qual fizeram parte 64 indivíduos que inicialmente foram avaliados por um ortopedista.

Posterior a essa avaliação, os participantes passaram por uma avaliação de triagem para o ingresso na Escola de Coluna que consistia em avaliação médica fisiátrica, avaliação fisioterápica, avaliação social, avaliação nutricional e avaliação psicológica. Após a realização de todas as avaliações, foram iniciadas as aulas somente para aqueles que apresentavam dor na coluna vertebral e haviam feito várias consultas médicas, tinham entre 18 a 60 anos e eram alfabetizados. Pacientes que apresentavam patologias de tratamento cirúrgico, neoplasias, cardiopatia, distúrbios pulmonares, crises epiléticas, distúrbios psiquiátricos, foram todos excluídos do programa. As aulas consistiam em 18 aulas com duração de dois meses (FURLAN et al., 1998).

Quanto aos conteúdos dessas aulas, foram trabalhados assuntos voltados à anatomia e fisiologia da coluna vertebral, as patologias que acometem a coluna e abordagens terapêuticas, benefícios e cuidados na execução dos exercícios, exercícios de alongamento, fortalecimentos, exercícios aeróbicos, e instrução para que os pacientes realizarem os exercícios em casa, ergonomia prática e importância do lazer.

A Divisão de Medicina de Reabilitação do Hospital das Clínicas - USP organizou sua escola de postura em setembro de 2001. O programa é formado por equipe multidisciplinar com profissionais da área de medicina, fisioterapia, psicologia, assistência social, terapia ocupacional, enfermagem, nutrição e educação física. Este programa tinha o objetivo de verificar a influência da patologia na qualidade 
de vida das pessoas (CESAR; BRITO JUNIOR; BATTISTELLA, 2004). Nesse trabalho, foi utilizado o questionário "Short Form Health Survey" (SF-36), questionário genérico auto-aplicável de qualidade de vida, que permite avaliar oito fatores: capacidade funcional, estado geral de saúde, aspectos físicos, dor, vitalidade, saúde mental e limitações dos aspectos sociais e emocionais. Este questionário foi validado para o Brasil, no ano de 1990 (FERRAZ et al., 1990).

O programa educacional e terapêutico foi oferecido durante quatro dias onde foram avaliados quatro momentos distintos: avaliação inicial, depois um e quatro meses contados da primeira avaliação.

Tsukimoto et al. (2006) estudaram a escola de postura desenvolvida pela divisão de Medicina de Reabilitação do Hospital das Clínicas da Faculdade de Medicina da USP (DMR).

O programa concedeu informações teóricas e prática e durante as aulas teóricas, foram abordados assuntos referentes à coluna vertebral, tais como: anatomia, reeducação alimentar, orientações quantoà postura correta na execução das atividades rotineiras e laboral, conscientização da importância da vida social, benefícios e cuidados durante os exercícios, e a importância da realização de alongamentos.

Inicialmente, os pacientes da escola de postura foram submetidos a uma triagem realizada pelo médico, psicólogo e assistente social. A equipe multidisciplinar foi formada por um médico, assistente social, psicólogo, fisioterapeuta, terapeuta ocupacional, enfermeiro, nutricionista e educador físico.

\section{Universidade Potiguar- Unp}

A escola de coluna da Universidade Potiguar, UnP, Natal, RN, ofereceu quatro aulas com freqüência de uma vez por semana, duração de sessenta minutos, coordenados por fisioterapeutas, divididas da seguinte forma (ANDRADE, 2005a).

Primeira aula: os indivíduos receberam noções de anatomia e fisiologia da coluna vertebral; fatores de risco para dor lombar; postura adequada durante o descanso e durante as atividades do cotidiano e laboral. Nas atividades práticas, os pacientes realizaram exercícios de alongamento dos músculos paravertebrais e fortalecimento dos músculos paravertebrais, flexores e extensores do quadril. Foram instruídos a realizarem a respiração do tipo padrão ventilatório diafragmático entre os exercícios.

Segunda e terceira aula: benefícios dos exercícios, patologias que acometem a coluna vertebral. Importância dos músculos paravertebrais e abdominais para a manutenção da postura adequada. Nas aulas práticas, repetiram-se os exercícios da primeira aula.

Quarta aula: revisão e ênfase na importância de continuarem seguindo as instruções recebidas.

\section{Universidade Federal De São Paulo (Unifesp)}

A escola de coluna no Centro de Reabilitação do Lar Escola São Francisco (UNIFESP) foi criado em 2003 para atendimentos dos pacientes com distúrbios vertebrais. Esse programa contou com uma equipe multidisciplinar composta por médico fisiatra, psicólogo, fisioterapeuta, terapeuta ocupacional e educador físico.

O procedimento metodológico adotado no programa Escola de Coluna consistia de quatro aulas teóricas oferecidas aos pacientes consecutivamente com duração de sessenta minutos coordenadas respectivamente pelo médico, terapeuta ocupacional, psicólogo e educador físico. Durante essas aulas, foram abordadas noções de anatomia e biomecânica da coluna vertebral; etiologia da dor na coluna e abordagem terapêutica; orientações posturais para a realização de atividades cotidianas e laborais; benefícios e a forma correta de executar a atividade física; a relação entre distúrbios na coluna vertebral e as alterações emocionais.

Após as quatro aulas teóricas, foram ofertadas as aulas práticas para grupos de dez indivíduos com duração de sessenta minutos, uma vez por semana. Nas aulas práticas os pacientes realizaram 
exercícios de alongamentos da cintura escapular, cintura pélvica e dos músculos dos membros inferiores; realizaram fortalecimento de glúteos, dos músculos abdominais e alguns grupos musculares dos membros inferiores; e estímulos para a correção postural por meio da conscientização das partes do próprio corpo. Os indivíduos foram orientados para persistirem realizando os exercícios domiciliares.

\section{Comparação Dos Principais Resultados Das Escolas De Postura}

Em todos os estudos houve uma participação bastante superior de pessoas do sexo feminino, exceto o trabalho publicado por Chung (1996) que não foram divulgados resultados divididos por sexo.

A média de idade dos participantes das escolas de posturas estudadas ficou entre 45 e 50 anos de idade, entre os quais podemos salientar o estudo de César, Brito Junior e Battistella (2004) que teve uma média de 44,5 anos, no estudo de Andrade (2005a), essa média foi de 44,8 anos, no de Caraviello et al. (2005) a média foi 48,1 anos e o estudo de Tsukimoto et al. (2006) que obteve uma média de idade de 46,8 anos.

Tabela 1. Distribuição dos estudos e seus referidos locais de execução com relação ao total de participantes e sexo.

\begin{tabular}{ccccccc}
\hline Estudo/Local & \multirow{2}{*}{ Total } & \multicolumn{3}{c}{ Masculino } & & \multicolumn{2}{c}{ Feminino } \\
\cline { 6 - 7 } & & $\mathbf{n}$ & $\mathbf{\%}$ & & $\mathbf{n}$ & $\mathbf{\%}$ \\
\hline Chung (1996) USP & 32 & $\mathrm{nr}$ & - & & $\mathrm{nr}$ & - \\
Furlan et al. (1998) USP & 64 & 12 & 18,8 & & 52 & 81,2 \\
Cesar, Brito junior e Battistella (2004) USP & 154 & 50 & 32,4 & & 104 & 67,6 \\
Tsukimoto et al. (2006) USP & 244 & 68 & 27,9 & & 176 & 72,1 \\
Andrade (2005a) UnP & 22 & 9 & 40,9 & & 13 & 59,1 \\
Caravielo et al. (2005) UNIFESP & 30 & 04 & 13,3 & & 26 & 86,7 \\
\hline
\end{tabular}

Tabela 2. Distribuição dos estudos e seus referidos locais de execução com relação à faixa etária estudada.

\begin{tabular}{cl}
\hline Estudo/Local & Faixa etária \\
\hline Chung (1996) USP & 18 a 70 anos \\
Furlan et al. (1998) USP & 18 a 60 anos \\
César (2004) USP & 16 a 75 anos \\
Tsukimoto et al. (2006) USP & 18 a 72 anos \\
Andrade (2005a) UnP & 18 a 60 anos \\
Caravielo et al. (2005) UNIFESP & 25 a 72 anos \\
\hline
\end{tabular}

Quanto aos resultados alcançados pelas escolas analisadas pelos referidos estudos, são bastante variados, no estudo de Chung (1996) os pacientes foram acompanhados durante um estágio na escola de coluna e reavaliados posteriormente em duas ocasiões, após 6 meses e um ano depois de terem participado da escola. Comparando o período anterior e posterior a participação na escola de postura, foi relatado que em $94 \%$ dos pacientes houve melhora da dor, e $66 \%$ desses pacientes continuaram a seguir as recomendações da escola de postura. 
No estudo de Furlan et al. (1998), após um ano da realização do curso, os pacientes foram reavaliados, dos 64 indivíduos que realizaram o curso 47 (78,3\%) compareceram para a reavaliação. Verificou-se, nessa reavaliação, que dos $81,2 \%$ dos pacientes que faziam uso de algum medicamento para a dor antes das aulas, apenas 18,8\% continuavam a fazer e entre os 57,5\% que estavam recebendo analgésicos, antiinflamatórios e/ou antidepressivos tricíclicos por orientação médica, após as aulas esse índice havia caído para 42,5\%. Quanto à intensidade da queixa dolorosa, foram reavaliados 42 através da escala analógica visual e em todos os casos tanto naqueles com cirurgia prévia, quanto naqueles sem cirurgia prévia houve uma redução considerável (acima de 50\%) da dor. Essa redução da dor refletiu diretamente também no numero de consultas médicas, que diminuiu de $50 \%$ antes do curso, para $26,2 \%$ após o curso.

Cesar (2004) relatou que os pacientes que fizeram parte de seu estudo, foram reavaliados em duas ocasiões posteriores as aulas, 1 e 4 meses depois, e foram verificadas melhoras significativas nos domínios de dor, estado geral de saúde, vitalidade e aspectos físicos. Já nos domínios de capacidade funcional, aspectos sociais, aspectos emocionais e saúde mental, não foram verificadas diferenças estatisticamente significativas.

Já Tsukimoto et al. (2006) relatam que foi feita nova avaliação depois de 1 mês, 4 meses e 1 ano. Devido a este longo período para a reavaliação, verificou-se uma perda de 54,9\% dos pacientes, restando apenas 110. Quanto aos resultados, houve melhora estatisticamente significante de todas as reavaliações em relação à avaliação inicial, mas não entre as reavaliações entre si, dos domínios Capacidade Funcional, Aspectos Físicos, Dor, Estado Geral da Saúde e Vitalidade. Por outro lado, o domínio Aspectos Sociais só apresentou melhora estatisticamente significante na segunda reavaliação, enquanto que Aspectos Emocionais só apresentou melhora estatisticamente significante na reavaliação após 1 ano.
No estudo de Andrade (2005a), verificou-se que $68,18 \%$ relataram redução na intensidade da dor, verificou-se que em $72,3 \%$ dos indivíduos houve melhora na incapacidade funcional e na avaliação da flexibilidade lombar houve melhora em $50 \%$ dos pacientes.

Os participantes da pesquisa realizada por Caraviello et al. (2005) apresentavam diferentes diagnósticos: $23(51,1 \%)$ apresentavam lombalgia mecânico postural, cinco $(11,1 \%)$ cervicalgia, quatro $(8,9 \%)$ lombociatalgia, dois $(4,4 \%)$ dorsalgia mecânico postural, quatro $(8,9 \%)$ tinham fibromialgia, três $(6,7 \%)$ osteoartrose da coluna e quatro $(8,9 \%)$ possuíam outras patologias como escoliose, artrite reumatóide, hipercifose, dorsal e antecedente de Mal de Pott.

Para a coleta de dados, foram utilizados instrumentos como questionário sócio demográfico, questionário de Roland Morris e EVA. Quanto aos resultados os 18 indivíduos $(60 \%)$ relataram melhora ao final do tratamento, oito $(26,7 \%)$ manifestaram piora e em quatro $(13,3 \%)$ pacientes não houve mudanças. Na avaliação da dor através da Escala Visual analógica de Dor, 17 (56,7\%) referiram melhora, seis $(20 \%)$ tiveram piora e sete $(23,3)$ não tiveram mudanças.

\section{Discussão}

É indiscutível que a intervenção da escola de postura tenha alcançado reconhecimento e popularidade, devido a sua efetividade terapêutica na abordagem da dor nas costas (CASAROTTO; MURAKAMI, 1995; HALL; ICETOM, 1983; VERSOLOOT et al., 1992). Porém a sua real efetividade ainda é discutida (ANDRADE, 2005b), pelo fato de que as escolas de postura vêm variando o número de aulas, número de participantes, tipo de profissionais envolvidos, práticas ou não de exercícios durante as aulas, modificando o método, ocasionando uma não eficácia do programa.

Em todos os relatos dos estudos trabalhados, verificou-se uma melhora significativa daintensidade 
de dor. Esses resultados possuem semelhança com outros programas de escola de postura, a exemplo do programa desenvolvido por Donchin et al. (1990) com 142 empregados de um hospital em Israel, e ele constou de 4 sessões de 90 minutos. Esse programa foi considerado como não efetivo no tratamento da lombalgia, mas outro exemplo considerado ineficaz, foi o trabalho desenvolvido por Daltroy et al. (1997), envolvendo 4000 trabalhadores postais. Neste estudo, foi aplicado a mesma metodologia que o anterior, mas não se verificou a redução das taxas de incidência de lombalgias, do custo médio do absenteísmo, nem das recorrências das queixas.

Por outro lado, a literatura nos traz evidências suficientes quanto à efetividade das escolas de postura. Assim como aquelas descritas anteriormente neste artigo (HALL; ICETON, 1983; CASAROTTO; MURAKAMI, 1995; INDAHL et al., 1998) em todos estes programas os resultados se mostraram positivos. Nachemsom (1992), reportou que a back school é altamente efetiva para lombalgia, cujos sintomas duravam entre 7 e 42 dias.

Além desses trabalhos relacionados, verificase que outras revisões de literatura mostraram a efetividade da escola de postura em várias situações. Entre eles, podemos salientar: Miralles (2001) que realizou estudo de revisão bibliográfica a respeito da metodologia das escolas de coluna. Observouse que há uma variação dependendo do local, e o autor analisou a escola de coluna da Suécia, Canadá, Califórnia, escola de coluna européia e espanhola, e em todas foram obtidos resultados positivos. Guzmán et al. (2001) realizou uma revisão bibliográfica acerca da eficácia da reabilitação, por meio da atuação multidisciplinar na lombalgia crônica, resultando em diminuição da dor e melhora funcional dos pacientes.

Alguns estudos de meta-análise como, por exemplo, o desenvolvido por Hall e Hadle (1995), avaliaram 6418 pacientes com dor na coluna e tratado em escola de postura, dos quais $64 \%$ dos pacientes afirmaram ter melhora na dor, e $98 \%$ disseram que o programa melhorou seus aspectos físicos e emocionais. Di Fábio (1995) também relatou resultados semelhantes aos de Hall e Hadle (1995), com melhora na dor e nos aspectos físicos e emocionais, em seu estudo prospectivo com 2713 pacientes com dor lombar, nos Estados Unidos.

\section{Conclusões}

Conclui-se, portanto, que todas as escolas de postura são utilizadas na prevenção secundária das afecções da coluna vertebral, sobretudo nas lombalgias, sendo estas uma importante ferramenta destapatologia. Verifica-se nas escolas analisadasque elas são bastante heterogêneas, pois há a existência de uma grande variedade em relação ao número de aulas, participantes, profissionais envolvidos e na metodologia das aulas práticas, somente algumas incluíram exercícios de fortalecimento.

Conforme relatado pelos trabalhos discutidos, tanto em nível nacional e internacional, a escola de postura é de fato um programa de resultados eficazes no tratamento das afecções da coluna, pois em grande parte dos estudos, os principais resultados referem-se à melhora da dor, da mobilidade da coluna e da incapacidade funcional.

\section{Referências}

ANDRADE, S. C.; ARAÚJO, A. G. R.; VILAR, M. J. P. "Escola de Coluna" na lombalgia crônica- implantação de um programa na Universidade Potiguar - UnP Natal- RN. Jornal da LIRNNE, Natal, v. 1, n. 2, p. 49-52, 2005a.

ANDRADE, S. C.; ARAÚJO, A. G. R.; VILAR, M. J. P. Escola de coluna: Revisão histórica e sua aplicação na lombalgia crônica. Revista Brasileira de Reumatologia, São Paulo, v. 45. n. 4, p 224-228, jul/ago. 2005b.

BRACCIALI, L. M. P.; VILARTA, R. Aspectos a serem considerados na elaboração de programas de prevenção e orientação de problemas posturais. Revista Paulista de Educação Física, São Paulo, v. 14, n. 2, 159-171, jul./ dez. 2000. 
CARAVIELLO,E.Z.; WASSERSTEIN, S.; CHAMLIAN, T. R.; MASIERO, D. Avaliação da dor e função de pacientes com lombalgia tratados com um programa de escola de postura. Acta Fisiatra, São Paulo, v. 12; n. 1; p. 11-14. 2005.

CESAR, S. H. K.; BRITO JUNIOR, C. A.; BATTISTELA, L. R. Análise da qualidade de vida em pacientes de Escola de Postura. Acta Fisiatra, São Paulo, v. 11, n. 1, p. 17-21, 2004.

CASAROTTO, R. A.; MURAKAMI, S. C. Grupo de Coluna e Back Skool. Revista de Fisioterapia, São Paulo, v. 2, n. 2, p. 65-71, 1995.

CHUNG, T. M. Escola de coluna - experiência do hospital das clínicas da Universidade de São Paulo. Acta Fisiátrica, São Paulo, v. 3, n. 2, p. 13-17, 1996.

DALTROY, L. H.; IVERSEN, M. D.; LARSON, M. G.; LEW, R.; WRIGHT, E.; RYAN, J.; ZWERLING, C.; FOSSEL, A. H.; LIANG, M. H. A Controlled Trial of an Education Program to Prevent Low Back Injuries. The New England Journal of Medicine, Andover, v. 337, n. 5, p. 322-328. 1997.

DI FÁBIO, R. P. Efficacy of comprehensive rehabilitation programs a back school for patients with low back pain: a meta analysis. Physical Therapy, Alexandria, v. 75, n. 10, p. 865-878, 1995.

DONCHIN M.; WOOLF, O.; KAPLAN, L.; FLOMANY. Secondary prevention of low-back pain. A clinical trial. Spine, Hagerstown, v. 15, n. 12, p. 1317-1320, 1990.

FERRAZ, M. B.; OLIVEIRA, L. M.; ARAÚJO, P. M. P.; ATRA, E.; TUGWELL, P. Cross-cultural reliability of the physical ability dimension of the health assessment questionnaire. The Journal of Rheumatology, Toronto, v. 17, n. 6, p. 813-817, 1990.

FORSSELL, M. Z. The back school. Spine, Hagerstown, v. 6, n. 1, p. 104-106, 1981.

FURLAN, A. D.; CASTRO, A. W.; CHUNG, T. M.; IMAMURA, S. T.; CAMANHO, G. L. Escola de Coluna-Programa desenvolvido no Instituto de Ortopedia e Traumatologia do HC/FMUSP. Acta Ortopédica Brasileira, São Paulo, v. 6, n. 2, p. 55-60, abr.jun. 1998.

GUZMÁN, J.; ESMAIL，R.; KARJALAINEN，K.; MALMIVAARA, A.; IRVIN, E.; BOMBARDIER, C. Multidisciplinary rehabilitation for chronic low back pain: systematic review. British Medical Journal, London, v. 322, n. 7301, p. 1511-1516, 2001.
HALL, H.; HADLER, N. M. Controversy in low back school. Education or exercise. Spine, Hagerstown, v. 20, n. 9, p. 1097-1098, 1995.

HALL, H.; ICETON, J. A. An overview with specific reference to the Canadian back education unit. Clinical Orthopaedics and Related Research, New York, v. 179, p. 11-23, 1983.

HEYMANS, M. W.; VAN TULDER, M. W.; ESMAIL, R.; BOMBARDIER, C.; KOES B,W. Back schools for nonspecific low back pain: a systematic review within the framework of the Cochrane Collaboration Back Review Group. Spine, Hagerstown, v. 30, n. 19, p. 2153-2163, 2005.

INDAHL, A.; HALDORSEN, E. H.; HOLM, S.; REIKERAS, O.; URSIN, H. Five-year Follow-up Study of a Controlled Clinical Trial Using Ligth Mobilization and an Informative Aproach to Low Back Pain. Spine, Hagerstown, v. 23, n. 23, p. 2625-2630, 1998.

MIRALLES, I. Prevención del dolor lumbar. Efectividad de la Escuela de Coluna. Revista de la Sociedad Española del Dolor, Madrid, v. 8, Supl. II, p. 14-21, 2001.

NACHEMSOM, A. L. Knewest knowledge of low back pain: a critical look. Clinical Orthopaedics, Philadelphia, v. 279 , p. 8-20, 1992.

SILVA, M. C.; NEIVA, A. C. G. F.; VALLE, C. J. Dor lombar crônica em uma população adulta do Sul do Brasil: Prevalência e fatores associados. Caderno de Saúde Pública, Rio de Janeiro, v. 20, n. 2, p. 377-385, mar/abr. 2004.

SNOOK, S. H. The design of manual handling tasks. Ergonomics, London, v. 21, n. 12, p. 963-85, 1978.

TEIXEIRA, M. J. Tratamento multidisciplinar do doente com dor. In: CARVALHO, M. M. M. J. (Org). Dor: um estudo multidisciplinar. São Paulo: Summus, 1999. p. 77-85.

TSUKIMOTO, G. R.; RIBERTO, M.; BRITO, C. A.; BATTISTELLA, L. R. Avaliação longitudinal da Escola de Postura para dor lombar crônica através da aplicação dos questionários Roland Morris e Short Form Health Survey (SF-36). Acta Fisiatra, São Paulo, v. 13, n. 2, p. 63-69, 2006.

VERSLOOT, J. M.; ROSEMAN, A. L.; VAN SON, A. M.; VAN AKKERVEC, P. F. The Cost-Effectiveness of a Back School Program in Industry. Spine, Hagerstown, v. 17, n. 1, p. 22-27, 1992. 